Horizons philosophiques

Horizons

\title{
Pietro Verri, théoricien milanais du bonheur ou La notion de rapidité résout-elle les problèmes liés au « bonheur froid et sec » de Maupertuis
}

\section{Roch Duval}

Volume 14, numéro 1, automne 2003

Au risque du bonheur

URI : https://id.erudit.org/iderudit/801249ar

DOI : https://doi.org/10.7202/801249ar

Aller au sommaire du numéro

Éditeur(s)

Collège Édouard-Montpetit

ISSN

1181-9227 (imprimé)

1920-2954 (numérique)

Découvrir la revue

Citer cet article

Duval, R. (2003). Pietro Verri, théoricien milanais du bonheur ou La notion de rapidité résout-elle les problèmes liés au « bonheur froid et sec » de Maupertuis. Horizons philosophiques, 14(1), 14-38.

https://doi.org/10.7202/801249ar d'utilisation que vous pouvez consulter en ligne. 


\title{
PIETRO VERRI, THÉORICIEN MILANAIS DU BONHEUR \\ OU \\ LA NOTION DE RAPIDITÉ \\ RÉSOUT-ELLE LES \\ PROBLÈMES LIÉS AU \\ «BONHEUR FROID ET SEC» \\ DE MAUPERTUIS.
}

\author{
Les philosophes s'accordent sur le bonheur, \\ comme sur tout le reste. \\ Julien Offray de La Mettrie'
}

Pietro Verri (1728-1797), philosophe milanais de l'époque des Lumières, a pris une part active aux innombrables discussions philosophiques prétendant générer une définition claire et précise du concept de bonheur. Suivant l'exemple de ses compatriotes italiens, Pietro Verri marche sur les brisées de Maupertuis (1698-1759) en commentant principalement la conception philosophique du bonheur élaborée par ce dernier. Bien qu'à l'instar de Maupertuis, Verri accorde une antériorité et une priorité absolue (tant qualitative que quantitative) à la douleur sur le plaisir, il juge quand même irrecevable la conception du bonheur de Maupertuis en taxant celle-ci de vaine paraphrase. Par conséquent, Verri propose une définition du plaisir (bonheur) qui se caractérise et se définit par une suppression "rapide" de la douleur. La notion de rapidité constitue le maître mot de cette définition et toute la validité de l'argumentation du philosophe lombard repose entièrement sur ce concept. En quoi l'introduction de la notion de rapidité constitue-t-elle vraiment une solution au problème philosophique du bonheur? Voilà la question que je désire aborder dans ce court texte.

Dans un premier temps, j'instruis le lecteur de l'importance générale d'une interrogation philosophique sur le bonheur dans la philosophie du XVIIIe siècle. Puis, dans un second moment, je brosse à grands traits la conception du bonheur proposée par Maupertuis, 
tout en prenant soin de retracer les grandes lignes de la réception de cette dernière en Italie, ceci dans le souci manifeste d'assurer une transition graduelle vers la solution proposée par Pietro Verri. S'ensuit un exposé général sur l'analyse du bonheur proposée par Pietro Verri dans le Idee sull'indole del piacere (Idées sur la nature du plaisir) (1773) - son texte le plus achevé en la matière - et, finalement, je tente de démontrer l'insuffisance de la solution verrienne en soumettant la notion de rapidité à un examen critique.

\section{Le bonheur, idée-force du XVIIle siècle.}

Avant d'aller inéluctablement s'échouer avec fracas sur le récif kantien ${ }^{2}$ - après avoir contourné, puis franchi non sans difficulté et au prix de maints efforts l'étoc rousseauiste - l'idée du bonheur constitue un de ces thèmes philosophiques sur lequel se sont échinés les plus grands esprits français du siècle des Lumières ${ }^{3}$. Du petit traité pratiquement méconnu de Formentin ${ }^{4}$ (1706), au début même de ce siècle propice en réflexions de toutes sortes, jusqu'au célèbre texte de Mme de Staël (1796) ${ }^{5}$, en passant chronologiquement par les œuvres phares de Fontenelle (1724) ${ }^{6}$, La Mettrie (1748) ${ }^{7}$, Maupertuis ${ }^{8}$ (1749), force est de constater que nulle autre époque de l'humanité - hormis peut-être la nôtre - n'a autant cherché à s'approprier, faire sienne, comprendre, analyser, décortiquer, puis propager l'idée du bonheur. Une telle profusion de traités, d'opuscules, de mémoires, d'essais, de pamphlets, souligne indiscutablement la "prégnance» de cette idée d'apparence toute simple dans la philosophie française. Or, comme le laisse sous-entendre la citation du philosophe et médecin malouin La Mettrie - apposée en exergue - une telle abondance trahit, révèle, le caractère protéiforme, voire insaisissable, de l'état d'esprit que l'on désigne d'une façon bien souvent maladroite par le mot «bonheur». Si les philosophes ne parviennent pas à s'accorder d'emblée sur l'analyse du terme «bonheur» c'est bien parce qu'avant tout sous ce vocable d'apparence si anodine se cache en vérité un concept se dérobant $a b$ initio à toute tentative d'analyse qui se voudrait simple. Entre, d'une part, les poncifs académiques de l'époque répétant ad nauseam la prétendue sagesse héritée de théories philosophiques rescapées de l'Antiquité, à savoir le triplet composé de la philosophie platonicienne, du stoïcisme, de l'épicurisme et, d'autre part, le foisonnement d'opuscules où des auteurs prétendument heureux, ou se targuant d'avoir atteint la félicité, se proposent de nous faire connaître leur recette pour parvenir à la béatitude, l'idée du bonheur 
- ce concept digne somme toute d'une véritable analyse philosophique - tombe finalement sous la coupe de véritables philosophes désirant soumettre celle-ci au sérieux d'une analyse digne de ce nom. Les récentes avancées de la science newtonienne9, conférant aux calculs mathématiques et à l'analyse dite géométrique une valeur prédictive indissociable du statut de méthode incontournable pour débusquer la vérité, puis l'émergence progressive d'une science dite morale, quoique aux fondements encore incertains, fera du concept du bonheur un thème sur lequel se pencheront les plus brillants esprits animés de ce nouvel esprit scientifique. Parmi les philosophes du XVIIIe siècle soucieux d'appliquer le sérieux de la méthode scientifique aux problèmes pérennes de la philosophie, le nom de Pierre Louis Moreau de Maupertuis se distingue avec maestria.

\section{L'Essai de philosophie morale de Maupertuis}

Parmi les traités philosophiques s'attelant à la tâche d'analyser le concept du bonheur, ceux de La Mettrie (1748) et de Maupertuis se démarquent avantageusement du reste de la production philosophique de l'époque. II est d'ailleurs singulier que ces deux philosophes d'origine malouine ${ }^{10}$ vivaient en exil à la cour de Frédéric II de Prusse. Ce fait particulier - une sorte de cosmopolitisme avant la lettre - tend à démontrer qu'au XVIII e siècle l'analyse philosophique du bonheur n'est pas uniquement un phénomène idoine à la philosophie française mais bien un thème qui intéresse l'ensemble de l'intelligentsia européenne ${ }^{11}$. Comme on le démontrera d'ailleurs ci-dessous, l'œuvre morale de Maupertuis trouvera écho en Italie et sera habilement commentée par un jeune lombard du nom de Pietro Verri. Mais avant de gloser sur les développements ultérieurs et extérieurs de la théorie du bonheur de Maupertuis, il se révèle impératif de présenter celle-ci, aussi bref et schématique cet exposé soit-il.

Les théories de La Mettrie (1709-1751) et de Maupertuis sont pratiquement indissociables dans la mesure où ce dernier rédige l'Essai de philosophie morale (1749) en réaction directe au traité de son compatriote, paru une année auparavant ${ }^{12}$. Si le contraste au niveau de la méthode est minime, car tous deux se targuent de suivre une méthode dite scientifique ${ }^{13}$ - en accordant la prééminence à une conception sensualiste tout en faisant table rase de toutes les conceptions métaphysiques brandissant le sceptre de la 
fiction éthérée d'une âme humaine pour l'un, et en proposant un calcul mathématique des plaisirs et des peines pour l'autre -, celui au niveau de l'esprit est saisissant. Alors que l'Anti-Sénèque de La Mettrie, exhortant à la licence, déborde de joie de vivre, d'audace, de pétulance, en prêchant d'une manière pratiquement scandaleuse un hédonisme libertin sans bornes ${ }^{14}$, le traité de Maupertuis est désolant, voire affligeant, par le pessimisme qui en exsude ${ }^{15}$. En effet, Maupertuis, cet être qui n'avait de passion que pour la science dit-on ${ }^{16}$, se relève avec peine de la tristement célèbre affaire König ${ }^{17}$ et, avec plus de componction, des railleries et de la cavale montée par son allié de jadis, Voltaire ${ }^{18}$. Ces tracas, se superposant à un caractère déjà peu enclin à l'exubérance, se font sentir, imbibent, imprègnent tout entier la prose de l'Essai de philosophie morale ${ }^{19}$. C'est donc en connaissance de cause que Maupertuis dira de son traité : «On l'a trouvé triste et sec : j'avoue qu'il l'est; mais je ne crois pas qu'il dut être autrement ${ }^{20}$ ». Examinons maintenant de quoi se compose ce traité.

Partisan avoué de la méthode more geometrico, Maupertuis dans un souci patent de clarté abat quelques cartes dès les premières lignes de son essai - toutefois sans montrer ab initio ses atouts en présentant tour à tour une définition des plaisirs et des peines, véritables pierres angulaires de sa théorie du bonheur. "J'appelle plaisir toute perception que l'âme aime mieux éprouver que ne pas éprouver. J'appelle peine toute perception que l'âme aime mieux ne pas éprouver qu'éprouver ${ }^{21}$ ». Définitions sèches, s'il en est, mais qui témoignent de la volonté analytique de son auteur. On trouve d'ailleurs ici en filigrane, un emprunt patent fait à La Mettrie. En effet, le médecin et philosophe malouin fait de la sensation physique (perception sensible), constituant l'essentiel de la vie physique et morale, le socle à partir duquel il échafaude une théorie du bonheur. Le plaisir et le bonheur ne seraient pas de nature différente; seule une différence de durée et de vivacité permettrait de les distinguer. La Mettrie écrit :

Nos organes sont susceptibles d'un sentiment ou d'une modification qui nous plaît et nous fait aimer la vie. Si l'impression de ce sentiment est courte, c'est le plaisir; plus longue, c'est la volupté; permanente, on a le bonheur. "C'est toujours la même sensation, qui ne diffère que par sa durée et sa vivacité22».

Or, il appert que l'idée de considérer le bonheur comme un état 
susceptible d'une mesure mathématique - auquel on peut appliquer les notions d'intensité et ou de durée - plut à l'esprit scientifique de Maupertuis car, dans la foulée de La Mettrie, il assoit également sa théorie du bonheur sur une base sensualiste ${ }^{23}$. C'est donc à partir de ces simples définitions introduites in limine que Maupertuis échafaude son système de philosophie morale. II est important d'observer ici que Maupertuis, suivant en cela John Locke, introduit une conception binaire ou dyadique des affections de l'âme : soit "plaisir" ou "peine»24. De fait, sans nier explicitement l'existence ontologique de perceptions indifférentes - c'est-à-dire de perceptions qui ne seraient ni de douleur ni de plaisir - Maupertuis élimine au niveau épistémique de telles perceptions indifférentes de son système de philosophie morale :

Je ne sais s'il y a des perceptions indifférentes, des perceptions dont la préférence ou l'absence soient parfaitement égales. Mais s'il y en a, il est évident qu'elles ne sauraient faire des moments heureux ni malheureux ${ }^{25}$.

Le temps que dure une perception nous procurant du plaisir se nomme un "moment heureux», tandis qu'un "moment malheureux» désigne le temps que dure une perception de peine (ou de douleur). En homme de science averti, Maupertuis s'appuie sur l'existence d'instruments de mesure éprouvés pour avancer l'idée qu'il est possible, sans risques de se tromper, de procéder à la mesure (objective) de la durée des perceptions de plaisirs ou de peines ${ }^{26}$. Toutefois pour que l'estimation des moments heureux ou malheureux soit complète, Maupertuis se doit de faire intervenir une unité de mesure supplémentaire, prenant en compte une quantité (subjective) selon laquelle on ressent un plaisir ou une peine. Cette nouvelle quantité de grandeur se nomme l'intensité. Ainsi, intensité et durée forment les deux unités de mesure à partir desquelles Maupertuis s'ingénie à comparer et à calculer les sensations de plaisirs et de peines.

Pour avoir l'estimation des moments heureux ou malheureux, il faut donc avoir égard non seulement à la durée, mais encore à l'intensité du plaisir ou de la peine. Une intensité double, \& une durée simple, peuvent faire un moment égal à celui dont l'intensité serait simple, \& la durée double. En général, l'estimation des moments heureux ou malheureux est le produit de l'intensité du plaisir ou de la peine par la durée $e^{27}$. 
Bien que le philosophe malouin reconnaisse qu'il n'existe malheureusement pas de manière exacte de mesurer l'intensité contrairement à la durée - il soutient néanmoins que l'existence d'un «jugement naturel» garantit la validité de la sensation subjective d'intensité28. Notons à ce propos que de nombreux critiques reprocheront ici à Maupertuis un manque de rigueur scientifique en soutenant que, puisque l'intensité repose sur un sentiment subjectif, elle rend inopérante toute tentative de comparaison (intersubjectivement valable) entre des plaisirs ou des douleurs.

Afin de poursuivre sa démonstration, Maupertuis introduit deux autres définitions. Ce sont les notions de bien et de mal : "Le bien est une somme de moments heureux. Le mal est une somme semblable de moments malheureux ${ }^{29}$ ". II en découle, si on poursuit l'argument more geometrico de Maupertuis, que le bonheur et le malheur se définissent par conséquent comme le résultat d'une opération mathématique dans laquelle intervient un calcul où l'on soupèse les valeurs respectives des biens et des maux. Cela étant posé, Maupertuis est finalement en mesure de proposer la définition suivante du bonheur : «Le bonheur est la somme des biens qui reste, après qu'on en a retranché tous les maux ${ }^{30}$ ». Puis, celle du malheur s'énonce comme suit : "Le malheur est la somme des maux qui reste, après qu'on en a retranché tous les biens ${ }^{31}$ ». Par conséquent, l'homme le plus heureux est celui qui, après la soustraction de la somme des maux, se retrouve avec la plus grande somme de biens. Dans le cas contraire, l'homme est malheureux. «Enfin ce n'est qu'après la déduction faite des biens \& des maux, qu'on peut juger du bonheur ou du malheur32".

Une fois cette constatation faite, il en découle que la tâche de tout homme soucieux d'atteindre le bonheur devrait être consacrée à connaître la nature même des biens et des maux afin soit d'accroître les biens, soit de diminuer les maux, ou bien de les comparer entre eux afin de faire le meilleur choix. La sagesse populaire semblerait ici nous conseiller de toujours préférer, volens nolens, le plus grand bien et d'éviter le plus grand mal. Or, ce subtil calcul, en apparence si simple, foisonne de difficultés. II y a d'une part le caractère hétérogène des objets comparés entre eux, puis il y a les difficultés engendrées par la comparaison d'un bien (mal) éloigné avec un bien (mal) présent. Mais la principale difficulté est la suivante, selon Maupertuis : 
II y a encore une autre comparaison plus difficile, \& qui n'est pas moins nécessaire : c'est celle du bien avec le mal. J'entends ici l'estimation du mal qu'il faudrait raisonnablement souffrir pour équivaloir à tel ou tel bien, ou l'estimation du bien dont il faudrait se priver pour éviter tel ou tel mal. Quoiqu'on ne puisse guère faire cette comparaison avec justesse, il y a une infinité de cas où l'on sent qu'il est avantageux de souffrir un mal pour jouir d'un bien, ou de s'abstenir d'un bien pour éviter un mal. Si les biens \& les maux sont vus dans différents éloignements, la comparaison devient encore plus difficile ${ }^{33}$.

Maupertuis donne le nom de prudence à cet art de faire adéquatement de tels calculs. On verra ci-dessous que Pietro Verri, inspiré en cela par la philosophie de Jean-Jacques Rousseau, incorporera à ce subtil art une dimension sociale, en faisant intervenir la vertu, perçue comme un fruit de la culture.

Sachant que le bonheur se réduit au résultat d'un calcul visant à comparer la somme des plaisirs avec celle des maux, Maupertuis est amené à réfléchir sur la quantité de biens et de maux qui se trouvent, en général, dans nos vies. Une constatation tombe comme un couperet: Que dans la vie ordinaire la somme des maux surpasse la somme des biens ${ }^{34}$. Voilà d'ailleurs ce qui expliquerait la propension des hommes à fuir la réalité, jugée morose, dans de nombreux divertissements dans lesquels on cherche l'oubli de soi et de l'existence en général35.

Dans le dessein de prouver sa thèse de la supériorité des maux sur les biens, Maupertuis introduit une distinction entre les plaisirs du corps et les plaisirs de l'âme ${ }^{36}$. II tente de démontrer, d'une part, que dans les plaisirs du corps la quantité de douleur excède la quantité de plaisir. II fera la même démonstration avec les plaisirs de l'âme. L'introduction d'une distinction entre les plaisirs de l'âme et ceux du corps et la reconnaissance de la différence dans la propriété des plaisirs qui en découlent sont deux moments dans la démonstration de Maupertuis qui seront critiqués par Verri. Pour comprendre la théorie de Verri il est nécessaire au préalable de résumer la position de Maupertuis.

Commençons par examiner les plaisirs du corps. Les plaisirs du corps ont ceci de particulier qu'ils diminuent en intensité plus on les ressent longtemps, tandis que la douleur augmente en intensité avec le temps ${ }^{37}$. II en résulte que l'on est susceptible de ressentir plus 
longtemps de la douleur que des plaisirs. Comme on le verra ci-dessous le philosophe italien Francesco Maria Zanotti contestera cette généralisation! Maupertuis juge également que seules quelques parties du corps peuvent nous procurer du plaisir, alors que toutes les parties du corps sont susceptibles de nous faire ressentir de la douleur. Maupertuis ne distingue malheureusement pas entre les parties du corps qui nous font directement ressentir de la douleur et celles, qui d'une manière diffuse, nous font éprouver de la douleur. Verri contournera cette imprécision en postulant l'existence de douleurs innommées (dolori innominati). Puis, finalement, Maupertuis postule que la quantité de plaisir que peut goûter notre corps est relativement petite ${ }^{38}$.

Maupertuis poursuit sa démonstration en examinant cette fois-ci les plaisirs de l'âme. Conscient que ce type de plaisir nécessite une définition précise, Maupertuis s'empresse de signifier clairement ce qu'il entend par plaisirs de l'âme :

...les plaisirs de l'âme se réduisent à deux genres de perception; l'un qu'on éprouve par la pratique de la justice, l'autre par la vue de la vérité. Les peines de l'âme se réduisent à manquer ces deux objets ${ }^{39}$.

Bien qu'il soit possible aux individus guidés par la sagesse (une vie vouée à défendre la justice et à poursuivre la vérité) d'espérer une vie où l'on constate une diminution de la somme des maux par rapport à celle des biens, cet état est uniquement le lot d'une minorité et de toute manière même les plus vertueux sont contraints à ressentir et à éprouver les plaisirs des sens.

Mais, me dira-t-on peut-être, les plaisirs de l'âme ne peuventils pas procurer aux hommes un sort plus heureux que celui que vous nous avez dépeint? N'y a-t-il donc pas des Sages dont la vie se passe dans la pratique de la justice, \& dans la contemplation de la vérité? Je veux croire qu'il y en a : mais outre les peines du corps auxquelles ils sont toujours exposés, si l'on compte les Aristides et les Newtons, on verra que ces hommes sont trop rares pour empêcher que la proposition ne soit vraie: Que dans la vie ordinaire la somme des maux surpasse la somme des biens ${ }^{40}$.

Ceci constitue la démonstration mathématique à laquelle Maupertuis soumet le calcul des plaisirs et des douleurs. La suite du 
traité se compose d'un examen des valeurs respectives de l'Épicurisme (voué à augmenter la somme des biens) et du Stoïcisme (consacré à diminuer la somme des maux). Tenant compte de ce qu'il a démontré auparavant, Maupertuis juge que le Stoïcisme constitue, à défaut de mieux, l'attitude la plus raisonnable à adopter. "Voilà jusqu'où la raison seule peut atteindre : voyons maintenant si la raison éclairée d'une nouvelle lumière peut aller plus loin; si elle peut nous enseigner des moyens plus sûrs pour parvenir au bonheur, ou du moins pour rendre notre condition meilleure ${ }^{41}$ ».

Or, selon Maupertuis, la religion chrétienne - cette vérité révélée - avec son message d'amour et d'espoir, est la voie que doivent adopter les hommes s'ils désirent vivre heureux. La "recette» du bonheur de Maupertuis se termine par un acte de foi dans lequel il exhorte les hommes à suivre leur cœur pétri des principes du christianisme 42 .

Dans la seconde moitié du XVIIIe siècle, l'œuvre de Maupertuis eut un certain retentissement en Italie. En effet, la traduction italienne de l'Essai de philosophie morale, habilement menée par Dorighi en 1756, suscita une vive réaction critique de la part des intellectuels italiens de l'époque. L'œuvre de Maupertuis, dès sa parution en France en 1749, avait déjà été scrutée à la loupe par de nombreux philosophes italiens. Mais la parution en 1756-57, chez l'éditeur vénitien Pietro Valvasense, d'un volume regroupant les différentes interventions italiennes à la théorie morale de Maupertuis, propulsa ce dernier à l'avant-scène et le fit connaître d'un vaste public italien ${ }^{43}$. Avec la philosophie morale de Maupertuis comme toile de fond, le calcul des plaisirs et des douleurs donna lieu à un échange corsé entre deux représentants respectés de la philosophie italienne du moment : Francesco Maria Zanotti (1692-1777) ${ }^{44}$ et le père Casto Innocente Ansaldi (1710-1780)45. Cependant, le débat tourne rapidement en une confrontation entre les valeurs respectives du Stoïcisme et de la religion chrétienne.

Zanotti, se décrivant lui-même comme un homme porté à la mélancolie et au caractère triste ${ }^{46}$, commenta l'œuvre de Maupertuis alors qu'il rédigeait son traité de morale péripatéticienne. L'ensemble de ses réflexions critiques à l'endroit de Maupertuis sont contenues dans Ragionamento del signor Francesco Maria Zanotti al conte Gregorio Casali sopra un libro francese del signore di Maupertuis intitolato : Essai de philosophie morale, un appendice au traité de morale mentionné ci-dessus ${ }^{47}$. Bien que Zanotti admirait l'œuvre 
scientifique de Maupertuis, l'idée de mesurer l'intensité et la durée du plaisir ou de la douleur sembla répugnante au philosophe et homme de science bolognais. Cependant la critique la plus sévère qu'il dirigea contre la théorie de Maupertuis concerne la nature de la douleur. Selon Zanotti, Maupertuis a erré en soutenant que la douleur augmente à mesure que le temps avance. Zanotti soutient que Maupertuis semble avoir ignoré ici le phénomène de l'habitude qui permet de s'adapter à la douleur à un point tel que l'on développe une sorte d'insensibilité.

Cependant le débat entre Zanotti et Ansaldi tourna rapidement à une confrontation entre la philosophie stoïcienne et la philosophie chrétienne ${ }^{48}$. Dès lors le débat prit une tournure théologique qui était étrangère à l'esprit même de la théorie de Maupertuis. À cette confrontation prirent part le père Pio Tommmaso Schiara ${ }^{49}$ - lequel donna raison à Zanotti contre Ansaldi en se rangeant sous l'autorité du premier, même si Ansaldi avait présumé trouver un allié naturel dans la personne de Schiara -, Giuseppe Antonelli et le comte Ludovico Barbieri. II faut cependant noter que la grande majorité de ces critiques contestaient l'idée même d'un calcul des plaisirs et de la douleur et concentraient plutôt leurs efforts à défendre ou proscrire un rapprochement entre le christianisme et la philosophie stoïcienne.

II faut attendre le philosophe et économiste vénitien Giammaria Ortes (1713-1790), désigné par d'aucuns comme l'épigone de Maupertuis en Italie, pour que la réflexion philosophique sur le calcul proprement dit des plaisirs et des douleurs revienne à l'avant-plan de l'interrogation philosophique. Ortes, animé du désir irrépressible de tout calculer ${ }^{50}$, apporta, en 1757, sa contribution aux théories philosophiques du bonheur dans un petit texte intitulé Calcolo de' piaceri e de' dolori della vita umana. (Calcul des plaisirs et des douleurs de la vie humaine $)^{51}$ en défendant l'attitude pessimiste de Maupertuis quant à la prévalence du mal sur le bien.

Lorsque Pietro Verri commence à rédiger ses réflexions philosophiques sur la nature du bien et du mal et le rapport que ceuxci entretiennent dans une philosophie du bonheur, les critiques italiennes dirigées contre la philosophie de Maupertuis circulaient librement en italien. À ce stade-ci, on peut conjecturer que la lecture des critiques a façonné l'approche de Verri52. II nous reste à examiner maintenant en quoi consiste l'approche de Verri. 


\section{Pietro Verri, théoricien milanais du bonheur.}

S'il est juste de dire qu'il existe un "paradoxe Maupertuis» c'est-à-dire un traité sombre sur le bonheur écrit par un homme porté vers la mélancolie, mais qui débouche, contre toute attente, sur une proclamation enthousiaste et lumineuse célébrant la force du cœur sur celle de la raison -, il ne serait pas moins faux de dire qu'il existe également un «paradoxe Verri». En effet, tout semble indiquer que les malheurs, les déceptions et les échecs cuisants qualifient la vie de Pietro Verri. À ce titre, son autobiographie fourmille de renseignements éloquents ${ }^{53}$. II y eut d'abord la présence d'une mère dominatrice et peu chaleureuse, les déboires avec Gabriele, père exigeant et peu conciliant, le forçant à se conformer au moule aristocratique auquel il appartenait 54 , puis les insuccès académiques et, finalement, un désir inassouvi d'être reconnu comme un auteur à part entière. Considérant ce parcours non dépourvu de revers de toutes sortes, il n'est guère étonnant que lors de la rédaction des Meditazioni sulla felicità (Méditations sur le bonheur), Verri ait écrit ceci à son grand ami Giambattista Biffi : "J'écris des pensées sur le bonheur, peut-être croyant de le trouver en arrangeant mes idées 55 ». C'est donc en homme triste que Verri médite sur le bonheur. En quoi les réflexions d'un homme si triste peuvent-elles nous apprendre quelque chose sur le bonheur?

Disons d'entrée de jeu, inspiré en cela par John Locke 56 , que Verri postule très tôt dans sa carrière littéraire, soit dès 1754 , la nécessité d'appliquer aux sciences morales la même rigueur que celle que l'on retrouve dans les sciences naturelles ${ }^{57}$. Le philosophe lombard délaissera pour un moment les réflexions sur l'économie et le commerce, ainsi que ses tentatives en vue d'introduire une réforme monétaire, pour se consacrer, dès 1763 , à un texte sur le bonheur. Les Meditazioni sulla felicità de 1763 connurent un certain succès; un succès qui se transporta même de l'autre côté des Alpes ${ }^{58}$. Puis, une dizaine d'années après, il traite de nouveau du problème du bonheur en l'abordant cette fois à partir des notions de plaisir et de douleur. La majorité des exégètes de la philosophie de Pietro Verri se sont concentrés sur l'analyse des différentes refontes des Meditazion 59 . Je considère, pour ma part, que les ldee sull'indole del piacere e del dolore (Idées sur la nature du plaisir et de la douleur) ${ }^{60}$ est le texte qui renferme le plus de matériel susceptible de nous donner des éléments pour répondre à Maupertuis. En outre, dans l'édition de 1781, Verri fait précéder son texte sur le bonheur (1763) de celui sur la nature 
des plaisirs et des douleurs (1773) en précisant que ce qu'il dit dans celui-ci sert de toile de fond à sa théorie du bonheur et conditionne la lecture. Puis, finalement, le philosophe milanais soutient avec un orgueil à peine dissimulé que la théorie des plaisirs et des douleurs qu'il propose dans les Idee constitue une véritable contribution à la philosophie ${ }^{61}$. En quoi consiste donc cette théorie si exceptionnelle?

Au départ, tout repose sur une simple constatation : le mal (la douleur) a une priorité ontologique, voire ontogénétique, sur le bien. Le bien (le plaisir) n'a aucune valeur positive en soi, il n'est que la suppression de la douleur. C'est la douleur, le manque, l'absence d'un objet convoité, la sensation d'un vide à combler, qui meut les êtres humains et qui est le moteur de la civilisation.

Je ne prétendrais pas que la douleur en soi est un bien, je dirais plutôt que le bien provient du mal, la stérilité produit l'abondance, la pauvreté fait naître la richesse, l'injustice suprême engendre le courage, en un mot la douleur est le principe moteur de tout le genre humain; elle est la cause de tous les mouvements de l'homme, lequel sans le concours de la douleur ne serait qu'un animal inerte et stupide et qui périrait, aussitôt né....62.

Pour étayer sa thèse, Verri fait intervenir dans l'introduction à l'édition de 1781 des alliés de taille, tels Platon63, Jérôme Cardan, Montaigne, John Locke64 et Lorenzo Magalotti (1637-1712). Mais c'est sans nul doute l'appel fait à une expérience personnelle - du temps où il était soldat à la guerre de Sept ans - qui constitue vraisemblablement l'élément déclencheur de sa réflexion sur la nature du plaisir et de la douleur (et, par extension, du bonheur).

Le tumulte de l'âme au cours d'une bataille est si grand, qu'après la victoire on voit des hommes parmi les plus insensibles et les plus endurcis verser des torrents de larmes de consolation. Larmes que le commun des mortels attribuerait à de l'affection pour leur prince, mais dans lesquelles le philosophe reconnaît l'effet de la suppression d'une peur violente jointe à l'idée des avantages personnels qu'ils espèrent acquérir avec ce nouveau degré de gloire65.

La reconnaissance, sentie in petto, de la prédominance de la douleur sur le plaisir se transforme rapidement en théorie philosophique lorsque Verri, dans l'orbe de John Locke emphatiquement désigné comme un luminoso genealogista (généalogiste brillant) -, se propose de se mettre en quête, d'analyser, de découvrir l'essence même des "deux principaux pivots" de la 
sensibilité humaine : le plaisir et la douleur66. Après avoir démontré l'insuffisance des définitions proposées par d'illustres prédécesseurs - en l'occurrence Descartes, Wolff et Sulzer - Verri s'attarde un instant sur la définition de Maupertuis en reconnaissant à celui-ci un avantage marqué sur ses précurseurs, mais il finit quand même par affirmer que, toute aussi séduisante que soit la définition de Maupertuis, elle ne fait guère mieux que de faire passer une paraphrase pour une définition 67 . Confronté à l'insuffisance des définitions antérieures, mais surtout assuré de la validité de la méthode introspective ${ }^{68}$, le philosophe milanais se propose par conséquent d'analyser ses propres sensations et de décomposer celles-ci afin de faire la lumière sur les propriétés respectives du plaisir et de la douleur.

Parmi les diverses sensations que nous ressentons, Verri distingue deux classes principales: les sensations physiques et les sensations morales 69 . Les sensations physiques sont celles qui sont causées par une action immédiate sur notre corps. Les sensations morales, quant à elles, sont celles qui ne proviennent pas d'une action directe et immédiate sur notre corps.

Les sensations physiques se subdivisent à leur tour en douleur et plaisir. Les douleurs physiques sont occasionnées par des déchirements (lacerazione) ou une sévère irritation (irritazione violenta) de notre corps, comme ceux que causent un coup violent (forte percossa), une coupure (taglio) ou une brûlure (abbruciamento).Les plaisirs physiques sont uniquement la suppression de tels effets sur notre corps. Les douleurs morales se distinguent des douleurs physiques en ceci qu'elles ne sont pas causées par un type particulier et précis d'action sur le corps. Comme exemple de douleurs morales, Verri nous propose de considérer le sentiment que nous éprouvons à l'annonce de la mort d'une personne qui nous est chère ou lorsque nous apprenons que nous venons de nous faire dévaliser. Pareillement, l'annonce d'un héritage insoupçonné ou bien le fait d'apprendre que l'on a été nommé à un poste prestigieux que l'on convoitait causent des plaisirs moraux. Ces plaisirs sont désignés comme tels, car là encore nous ne pouvons isoler une action directe sur le corps.

Tous les hommes, même les plus frustres et sauvages, sont susceptibles d'éprouver des douleurs physiques, tandis que les douleurs morales sont l'apanage de gens ayant atteint un certain 
niveau de conscience. "Les plaisirs et les douleurs morales sont d'autant plus grands qu'est grand le nombre des besoins et des relations qui unissent les hommes entre eux ${ }^{70}$ ». On voit poindre ici l'idée que le bonheur est davantage une question qui concerne les hommes vivant en société, façonnés par une culture, qu'une question se rapportant uniquement aux individus isolés. Verri, le moraliste est avant tout un économiste qui prépare les mentalités à recevoir l'idée de l'utilitarisme. II existe d'ailleurs in nuce une conception utilitariste tant chez Verri que chez son grand ami et collaborateur Cesare Beccaria. Cependant, pour les fins de ce texte, nous nous contentons uniquement ici d'analyser la conception des plaisirs et des douleurs dans leur dimension épistémique.

Insatisfait de laisser la définition des sensations morales sans principe directeur, Verri examine une série d'exemples de douleurs et de plaisirs moraux pour en extraire des éléments communs permettant d'en dégager une caractéristique commune. La conclusion à laquelle parvient Verri est péremptoire : "Toutes nos sensations de plaisir ou de douleur dépendent uniquement de trois principes : une action immédiate sur le corps, l'espoir et la crainte. Le premier principe cause toutes les sensations physiques, les deux autres les sensations morales ${ }^{71}$ ». Verri poursuit sa démonstration en nous disant cette fois-ci que les plaisirs moraux naissent toujours de l'espoir (esperanza) de combler l'absence d'un bien douloureusement ressenti comme un manque (difetto), une imperfection. Impossible donc de ressentir un plaisir moral si on ne suppose pas l'existence antérieure d'un mal, d'un manque faisant obstacle à notre bonheur. Considérant que cela est suffisant pour justifier la thèse selon laquelle un plaisir moral est toujours précédé d'un mal moral, Verri nuance son analyse en apportant la précision suivante : s'il est vrai que le plaisir moral s'accompagne toujours d'une suppression de la douleur, il est également vrai de dire que tous les cas de suppression de la douleur n'engendrent pas tous des plaisirs moraux. Pour comprendre cette subtilité, le philosophe italien nous demande de considérer deux exemples.

Imaginons dans un premier temps, un individu, follement amoureux de son épouse, à qui l'on annonce sans ménagement que cette dernière vient de contracter une maladie mortelle s'accompagnant d'une douloureuse agonie. À l'annonce de la funeste nouvelle, l'époux éploré est en proie à un véritable tourment (strazio), 
il éprouve, dirons-nous, une véritable douleur morale. Puis, supposons que contrairement au pronostic des médecins, la maladie de la femme - quoique toujours gravement malade au départ évolue d'une manière telle que graduellement elle retrouve la santé, après une période de convalescence.

Supposons maintenant un scénario semblable, mais à la seule différence que dans l'exemple présent l'époux, pleurant l'annonce inopinée de la maladie de sa femme, voit soudainement la porte de sa demeure s'ouvrir pour livrer le passage à son épouse. L'annonce de la maladie de sa femme n'était qu'une blague de mauvais goût, car cette dernière est en parfaite santé.

Verri juge différemment ces deux exemples. Selon lui, dans le premier cas, l'époux n'éprouve pas de plaisir moral à la guérison de sa femme car cette guérison fut graduelle et lente. Le passage entre l'état initial de choc (l'annonce de la maladie) et celui terminal (la guérison) se fait tellement graduellement que l'esprit du mari s'habitue à la lente guérison de sa femme sans éprouver le moindre plaisir lorsque survient sa guérison. La suppression de la douleur fut tellement lente que la guérison de la patiente ne procura aucun plaisir moral au mari. Mais, dans le second cas, l'homme ressent un vif plaisir moral car la suppression de la douleur fut rapide. C'est à partir d'un tel exemple que Verri introduit sa thèse principale : le plaisir est une suppression rapide de la douleur :

Le plaisir naît donc de la douleur et consiste dans la suppression rapide de celle-ci et le plaisir est d'autant plus grand qu'est grande la douleur et qu'est rapide la disparition de celle-ci. Plus on en diminue la rapidité, plus on en diminue la sensation agréable72.

Le même raisonnement est appliqué aux plaisirs physiques : la plupart de ceux-ci sont occasionnés par une suppression rapide de la douleur73.

S'il y a plaisir (moral ou physique) uniquement lorsqu'il y a une suppression rapide de la douleur, alors comment doit-on nommer l'état dans lequel on se trouve si la suppression ne se fait pas rapidement? II semble que Verri ne donne pas de nom à cet état que l'on pourrait qualifier de neutre. On se rappelle qu'au début de son exposé, Verri a réduit les sensations à deux classes : les plaisirs et les douleurs. Comment peut-il maintenant postuler l'existence d'une sensation qui ne serait ni de la douleur, ni du plaisir? Verri demeure 
flou à ce sujet. II en découle, par conséquent, que Verri prend position quant à l'existence d'un état neutre (composé ni de douleur, ni de plaisir), état dont Maupertuis déclarait ignorer l'existence. L'introduction d'un tel état neutre joue un rôle précis dans la théorie de Verri. En vérité c'est ce qui lui permet d'affirmer que la somme des maux (douleurs) surpasse toujours celle des biens, car s'il existe une quantité de mal qui ne trouve pas de compensation dans une quantité de bien, alors en fin de compte la somme des maux (douleur) sera toujours supérieure à celle des biens (plaisirs).

Bien que la théorie de Verri soit fort complexe et fouillée, nous nous limitons dans le cadre de cet article à exposer ces quelques éléments de sa théorie. Contentons-nous de répéter qu'en soutenant que le plaisir se définit uniquement comme la suppression rapide de la douleur, Pietro Verri considère sa définition nettement supérieure à celle de Maupertuis. Mais qu'en est-il vraiment?

$\mathrm{Si}$, d'une part, il est vrai que la théorie de Verri permet de comprendre comment la somme des sensations de douleur peut surpasser celle des sensations de plaisir (car les douleurs qui se suppriment lentement n'engendrent pas de plaisir), il n'en demeure pas moins que l'argument qu'il utilise est fallacieux. En quoi la rapidité de la suppression d'une douleur peut-elle qualifier la nature du plaisir?

Contentons-nous pour l'instant de distinguer la durée (principe à la fois reconnu et admis dans la théorie de Maupertuis et dans celle de Verri) de la rapidité. On peut admettre que les «moments de plaisir» ou les «moments de douleur» (pour utiliser la terminologie de Maupertuis) ou les sensations de douleur ou celles de plaisir (pour utiliser la terminologie de Verri) ont une durée; c'est-à-dire qu'il est possible de mesurer celles-ci à l'aide d'un instrument de mesure (une montre ou un chronomètre, par exemple) donnant une valeur objective de leur durée. La durée d'un phénomène constitue une propriété que nous pourrions qualifier d'intrinsèque à ce phénomène. Une sensation de douleur, du moment qu'elle est perçue par l'esprit possède nécessairement une durée; elle a un commencement, une origine et une fin $^{74}$. Bien que l'attribution d'une durée à des phénomènes sensitifs ou émotifs ne soit pas exempte de problèmes conceptuels ${ }^{75}$, on peut néanmoins s'entendre sur le fait qu'ils sont quand même mesurables d'une façon objective (une fois que l'objet devant être mesuré est bien défini). Or, la rapidité ne constitue nullement une propriété temporelle qui est intrinsèque aux phénomènes. La rapidité désigne plutôt une propriété relative ${ }^{76}$. En ce 
sens ce type de propriété ne peut jamais être invoqué pour prouver l'existence de quelque chose; seule une propriété intrinsèque est investie de ce pouvoir. Lorsque je parle d'une suppression rapide de la douleur cela présuppose que le qualificatif «rapide» établit uniquement une comparaison avec une suppression qui aurait une autre durée (une durée plus longue), mais je ne peux me servir de ce qualificatif pour établir l'identité d'un phénomène. Ainsi, lorsqu'on parle d'une suppression rapide, il faut donc toujours se demander ceci : rapide par rapport à quoi?

De plus si l'on désire attribuer la propriété de la rapidité à un phénomène nous devons être en mesure d'établir des critères nous permettant de distinguer ce qui est rapide de ce qui ne l'est point. Vraisemblablement, Verri ne fait pas cela. II se contente uniquement de dire que le plaisir provient d'une suppression rapide de la douleur, sans jamais nous expliquer ce qu'est la rapidité ou nous donner des moyens (des critères précis) nous permettant de savoir si une suppression est rapide ou ne l'est pas. En l'absence de tels critères, la définition de Verri semble tout à coup bien inutile.

De toute manière, même si nous supposions que la rapidité désigne une propriété nous permettant de saisir les propriétés essentielles de la douleur et du plaisir, la définition de Verri, telle qu'elle se présente demeurerait tout de même contre intuitive. Reprenons à nos frais le célèbre exemple de l'opération de Cheselden sur l'aveugle de naissance Saunderson. II ne fait aucun doute ici que l'aveugle retrouvant soudainement la vue n'éprouvera aucun plaisir. Or, ici le passage de la cécité totale à la vision se fait rapidement. II suffit de quelques coups habiles de bistouri pour passer de l'obscurité la plus complète, de la privation de la vision (un mal en soi) au plein exercice de cette faculté. Malheureusement on connaît trop le cas Saunderson pour savoir combien l'acquisition de la vision fut douloureuse. La douleur fut également morale, car cet habile mathématicien dut également patiemment réapprendre à identifier visuellement les objets dans l'espace; objets qu'il identifiait auparavant à l'aide de sensations haptiques. Peut-on parler dans le cas de Cheselden d'une suppression rapide de la douleur n'engendrant aucun plaisir? Combien d'autres exemples de ce type nous faut-il pour douter de la validité de la définition proposée par Verri? 


\section{Conclusion}

Le calcul arithmétique des plaisirs et des douleurs de Maupertuis constitue une étape importante dans l'histoire des théories du bonheur. Cette théorie fut largement critiquée par un très grand nombre de philosophes. Parmi ceux-ci se trouve un philosophe milanais du nom de Pietro Verri. Bien que ce dernier partage avec Maupertuis une vision du monde où la somme des maux (douleurs) surpasse toujours celle des biens (plaisirs), il conteste néanmoins la définition du bonheur proposée par le philosophe malouin. Préférant l'analyse fine des sensations aux calculs abstraits, Verri propose une définition disant que le plaisir (bonheur) est engendré par une suppression rapide de la douleur. Tout aussi intéressante qu'elle soit, cette définition n'en est pas moins insuffisante, voire fautive. La rapidité ne constitue pas une propriété essentielle et intrinsèque des phénomènes, mais, tout au plus, elle en constitue une propriété relative. De telles propriétés ne nous sont d'aucun secours pour identifier un phénomène. De plus, s'agissant d'une propriété relative, Verri ne propose aucun critère pour juger objectivement de la «rapidité» d'un phénomène. Quand sait-on si un phénomène est rapide ou non? En l'absence d'un tel critère la notion de rapidité est bien inutile. Finalement je propose comme contre-exemple à la théorie de Verri le cas de l'aveugle Saunderson. Pour conclure, nous pourrions dire que le seul rôle que joue la rapidité dans la démonstration de Verri, c'est de servir d'explication à l'idée que la somme des maux surpasse toujours celle des biens. Voilà une bien triste consolation pour une idée que son auteur voulait si lumineuse et brillante! C'était peut-être croire trop rapidement aux vertus de la rapidité... L'homme heureux n'est-il pas après tout celui qui sait être patient77.

\section{Roch Duval Université de Montréal}

1. Julien Offray de la Mettrie, Anti-Sénèque ou Le souverain bien, dans De la volupté : Anti-Sénèque, ou Le souverain bien, L'École de la volupté, Système d'Épicure; édition préfacée, établie et annotée par Ann Thomson, Paris, Éditions Desjonquières, 1996, p. 27. (Collection XVIII ${ }^{e}$ siècle).

2. “La raison n'est apte qu'à saisir des idées épurées de toute contingence affective. Le bonheur doit se vivre, et il se volatilise, si on l'isole du cours irrationnel de l'existence. Kant saura tirer de cette contradiction une conclusion définitive : la recherche du bonheur ne peut pas être le fondement de la morale, parce qu'elle est 
sans commune mesure avec la raison. Le bonheur est un simple «idéal» de "l'imagination", une "réalité empirique», non un «impératif catégorique». Robert Mauzi, L'ldée du bonheur dans la littérature et la pensée française au XVIIIe siècle. Paris, Librairie Armand Colin, $4^{e}$ édition, 1969, p. 97. Le locus classicus de la disqualification kantienne de l'idée du bonheur comme fondement de la morale se trouve dans le Fondements de la métaphysique des mœurs (1785).

3. Bien que la question philosophique du bonheur soit née avec la philosophie elle-même, il n'en demeure pas moins que la philosophie française du XVIIIe constitue une période d'or où la discussion philosophique sur le bonheur était une activité particulièrement florissante. On dénombre une cinquantaine de traités ou petits ouvrages, de qualité variable, ayant pour thème le bonheur. Pour un aperçu historique de la question du bonheur en philosophie, de l'Antiquité grecque à aujourd'hui, voir l'article «Glück, Glückseligkeit» dans le Historisches Wörterbuch der Philosophie, Joachim Ritter (éd.), Bâle, Stuttgart, 1971, p. 680-707. Pour une vue d'ensemble synoptique concernant exclusivement la philosophie française, la lecture de l'ouvrage désormais classique de Robert Mauzi s'impose. Voir Mauzi, op. cit.

4. M. F. Formentin, Traité du bonheur, Paris, J. Guilletat, 1706.

5. Madame de Staël, De l'influence des passions sur le bonheur des individus et des nations : suivi de Réflexions sur le suicide; préface de Chantal Thomas, Paris, Payot \& Rivages, 2000. (Rivages poche/Petite bibliothèque).

6. Bernard le Bouyer de Fontenelle, Discours sur le bonheur, Paris, M. Brunet, 1724.

7. La Mettrie, "Anti-Sénèque...", op. cit.

8. Pierre-Louis Moreau de Maupertuis, Essai de philosophie morale. Berlin, 1749.

9. L'idée d'un parallélisme entre la loi newtonienne de la gravitation et une science du bonheur est esquissée par Delisles de Sales (1739?-1816) (pseudonyme de JeanBaptiste-Claude Izouard). "...L'homme bien organisé gravite vers le bonheur, comme les corps pesants vers le centre du globe...", Delisles de Sales, De la philosophie du bonheur, Paris, 1796, T. 1, p. 25.

10. À propos de cette origine commune, Saint-Malo, Maupertuis a écrit : "Nous sommes de la même ville. Cette raison seule aurait suffi pour que je lui voulusse du bien". Maupertuis, CEuvres, 1756, tome III, p. 344. Maupertuis attire également à Berlin un autre malouin célèbre, l'abbé Nicolas-Charles-Joseph Trublet (16971770).

11. II suffit uniquement de mentionner, entre autres, le nom de l'Allemand Johann Georg Sulzer (1720-1779). Quant à l'émigré d'origine française Jean Henri Samuel Formey (1711-1797), professeur de philosophie au Collège français à Berlin et secrétaire perpétuel de l'Académie royale des sciences et belles-lettres de Prusse, il appartient tant à la philosophie française qu'à la philosophie allemande. Outre les ouvrages italiens que nous allons citer plus bas, celui de Ludovico Antonio Muratori, Della pubblica felicità [Du bonheur public] (1749) est digne de mention.

12. Le texte sur le bonheur de La Mettrie parut à Potsdam en 1748. David Beeson, s'appuyant sur la correspondance de Maupertuis, conjecture que c'est vers le mois de mai 1749 , en revenant tout juste de la France, que Maupertuis prit connaissance du texte de La Mettrie et qu'une réplique était prête dès l'automne 1749. Cette première mouture, qui n'était pas destinée à être publiée, fut publiée à son insu vers la fin du mois de novembre 1749 ou au début de décembre 1749. Soucieux de 
Pietro Verri, théoricien milanais du bonheur ou La notion de rapidité résout-elle les problèmes liés au "bonheur froid et sec" de Maupertuis

présenter un ouvrage un peu plus soigné, afin d'éliminer des maladresses dans l'édition piratée, Maupertuis remania le texte et il en envoya une copie corrigée à l'abbé Sallier, le 15 septembre 1750. Voir David Beeson, Maupertuis : an intellectual biography, The Voltaire Foundation, Oxford, 1992, p. 193-194. (Studies on Voltaire and the Eighteen Century. 299).

13. «...pour expliquer le mécanisme du bonheur, nous ne consulterons que la nature et la raison, les seuls astres en effet capables de nous éclairer et de nous conduire,..." La Mettrie Anti-Sénèque, p. 29 L'argument scientifique de Maupertuis est toutefois subordonné à une apologie de la religion chrétienne.

14. Le philosophe grec Panajotis Kondylis considère d'ailleurs que l'Anti-Sénèque de La Mettrie constitue un des hauts lieux de la philosophie des Lumières. Voir Panajotis Kondylis, Die Aufklärung im Rahmen des neuzeitlichen Rationalismus (Les Lumières dans le cadre du rationalisme moderne), Stuttgart, Keltt-Cotta. 1981. (Ouvrage réédité en 1986, dtv-TB, puis chez Felix Meiner en 2002).

15. Voir de Corrado Rosso, "La triste felicità di Maupertuis e i suoi echi in Italia» (Le bonheur triste de Maupertuis et ses échos en Italie) dans Corrado Rosso, Illuminismo, felicità, dolore. Miti e ideologie francesi (Lumières, bonheur, douleur. Mythes et idéologies françaises). Naples, Edizioni scientifiche italiane, 1968, p. 27-41.

16. C'est ce qui explique le caractère très dépouillé du style littéraire de Maupertuis. Une écriture sèche, sans fioritures de style, ni embellissements inutiles, toute vouée au seul but qu'il s'est fixé : convaincre le lecteur en utilisant uniquement des arguments rationnels. Maupertuis, le savant, a joué un rôle actif en contribuant, entres autres, aux développements des Sciences Mathématiques, de la Biologiste, de l'Astronomie, de l'Embryologie, de la Géographie, de la Physique. Maupertuis laissa également son nom comme celui qui mesura en Laponie septentrionale un méridien terrestre. Voir de Mary Terrall, The Man who flattened the Earth. Maupertuis and the Sciences in the Enlightenment. Chicago, The University of Chicago Press, 2002.

17. Cette querelle concerne la priorité de la découverte du uprincipe de la moindre action".

18. Voltaire prend un malin plaisir à ridiculiser son ancien ami dans un écrit intitulé Diatribe du Docteur Akakia, Médecin du Pape (1753).

19. Les citations tendant à démontrer que Maupertuis était un être foncièrement triste, voire atrabilaire, sont légion. Voltaire ridiculise son adversaire en disant "Nous nous engageons à ne plus écrire tristement sur le bonheur...". Frédéric II, pourtant son protecteur, écrit ceci de la théorie du bonheur : "Mais vous ne m'avez pas persuadé que je fusse malheureux. J'ai vu seulement avec chagrin que vous l'étiez..." Cités dans Corrado Rosso, Illuminismo..., p. 30. D'autre part, Montesquieu écrit : «M. de Maupertuis, qui a cru toute sa vie et qui peut-être a prouvé qu'il n'était pas heureux, vient de publier un petit écrit sur le bonheur. Montesquieu, Correspondance, t. II, p. 235, lettre 480. Puis Mme de Puisieux écrit : «Parcourez le Traité du Bonheur de Fontenelle et vous prononcerez, malgré vous, que l'écrivain était heureux. Vous sortirez moins éclairés peut-être de son ouvrage que de l'Essai de philosophie morale de Maupertuis ; mais vous en sortirez plus content. Vous aimerez mieux la vie après avoir lu Fontenelle; après avoir lu Maupertuis, vous voudriez presque être mort. Si j'osais, je dirais que l'un présente surtout des bonbons qui fondent délicieusement dans la bouche, et que l'autre met sous la dent 
des noisettes qui sont dures à casser et qui ne donnent quelquefois que de la poussière. Demandez à Fontenelle ce que c'est que le plaisir et ne craignez pas qu'il vous réponde que c'est en général toute perception que l'âme aime mieux éprouver que de ne pas éprouver. Quelle triste définition du plaisir!...Je trouve que M. de Maupertuis a prétendu soumettre tout le monde à une arithmétique morale qui lui est propre et appliquer à tous les hommes un calcul qui ne convient qu'à ceux de sa classe. Mme de Puisieux, Londres, Les Caractères, 1750, p. 173.

20. Maupertuis, CEuvre, tome 1, Préface, p. 190-91.

21. Maupertuis, Essai de philosophie morale, p. 194

22. La Mettrie, Anti-Sénèque...p. 29. Mon souligné.

23. Bien que partageant la même base sensualiste que La Mettrie, Maupertuis ne nie pas l'existence de l'âme : "Les impressions des objets sur nos corps sont des sources de plaisir \& de peine : les opérations de notre âme en sont d'autres. Et tous ces plaisirs, \& toutes ces peines, quoiqu'entrées par différentes portes, ont cela de commun, que ce ne sont que des perceptions de l'âme, dans lesquelles l'âme se plaît ou se déplaît, qui sont des moments heureux ou malheureux». Maupertuis. Essai, p. 206

24. John Locke dans l'Essay on Human Understanding écrit, en parlant du plaisir et de la douleur : "Ce sont les pivots sur lesquels roulent toutes nos Passions". La citation de Locke provient de la traduction de M. Coste : Essai philosophique concernant l'entendement humain, J. Scheurer et P. Mortier le Jeune, Amsterdam et Leipzig, 1754, p. 454.

25. Maupertuis. Essai....lbid., p. 194.

26. "On peut aisément comparer les durées; nous avons des instruments qui les mesurent, indépendamment des illusions que nous pouvons nous faire". Maupertuis, ibid., p. 195.

27. Maupertuis, ibid., p. 196.

28. "Mais quoique nous n'ayons pas de mesure exacte pour les intensités, nous sentons bien que les unes soient plus grandes que les autres, \& nous ne laissons pas de les comparer. Chaque homme, par un jugement naturel, fait entrer l'intensité \& la durée dans l'estimation confuse qu'il fait des moments heureux ou malheureux." Maupertuis, ibid., p. 196.

29. Maupertuis, ibid., p. 197.

30. Maupertuis, ibid., p. 197.

31. Maupertuis, ibid., p. 197.

32. Maupertuis, ibid., p.198.

33. Maupertuis, ibid., p. 199.

34. Maupertuis, ibid., p. 203.

35. On retrouve ici une rémanence de la critique et de la condamnation pascalienne des divertissements.

36. II est important de souligner ici que Maupertuis ne considère nullement que les plaisirs du corps sont inférieurs aux plaisirs de l'âme. Ce sont tous deux des perceptions qui se passent dans l'âme.

37. «En examinant la nature des plaisirs \& des peines du corps, nous commencerons par une remarque bien affligeante : c'est que le plaisir diminue par la durée, \& que 
Pietro Verri, théoricien milanais du bonheur ou La notion de rapidité résout-elle les problèmes liés au «bonheur froid et sec" de Maupertuis

la peine augmente. La continuité des impressions qui causent les plaisirs du corps en affaiblit l'intensité : l'intensité des peines est augmentée par la continuité des impressions qui les causent». Maupertuis, ibid., p. 208.

38. "Enfin il y a une autre considération à faire. Le trop long, ou le trop fréquent usage des objets qui causent les plaisirs du corps conduit à des infirmités : \& l'on n'en devient aussi que plus infirme par l'application continuée ou répétée trop souvent des objets qui causent la douleur. II n'y a ici aucune espèce de compensation. La mesure des plaisirs que notre corps nous peut faire goûter est fixée \& bien petite; si on y verse trop, on en est puni : la mesure des peines est sans bornes, \& les plaisirs même contribuent à la remplir». Maupertuis, ibid., p. 210-211.

39. Maupertuis, ibid., p. 212.

40. Maupertuis, ibid., p. 214

41. Maupertuis, ibid., p. 232.

42. Dans un vocabulaire kantien, nous pourrions dire que le cœur acquiert une primauté pratique sur la raison.

43. Raccolti di Trattati di diversi Autori concernenti alla Religione Naturale e alla Filosofia Morale dei Cristiani e degli Stoichi. Venise, Pietro Valvasense, 1756-1757.

44. Ce jésuite fut l'un des premiers, à Bologne au début des années 1720, à soumettre la théorie des couleurs de Newton à un examen critique et expérimental (à l'aide d'un spath d'Islande comme prisme). Outre son activité scientifique on lui doit des ouvrages de philosophie morale et d'esthétique d'une grande qualité. Parmi cellesci nous pouvons nommer La forza attrativa delle idee (1747), La filosofia morale secondo i peripatetici (1754), Dell'arte poetica (1758).

45. Son ouvrage le plus connu est Riflessi sopra i mezzi di perfezionare la filosofia morale. Édition critique dirigée par A. Rizzacasa et R. Gatti, Naples, 1987 [Pubblicazioni dell'Universita degli studi di Perugia]

46. Voir Giovanni Fantuzzi, Notizie della vita e degli scritti di Francesco Maria Zanotti, Stamperia di San Tomasso d'Aquino, Bologne, 1778. p. 54-55. Je tire cette référence de l'ouvrage de Franco Venturi, Settecento riformatore. Da Muratori a Beccaria. Guido Einaudi, 1969, p. 396.

47. Francesco Maria Zanotti, Filosofia morale secondo l'opinione dei peripatetici ridotta in compendio (1754) (Giambattista Novelli, Venise, 1763).

48. À ce propos voir de Casto Innocente Ansaldi, Vindicia Maupertuisianae, traduit en Italien sous le nom de Difesa del Signor di Maupertuis dalle Censure del Signor Dottore Francesco Maria Zanotti.

49. Directeur de 1746 à 1759 de la bibliothèque Casanatense à Rome.

50. Dans une formule lapidaire, Italo Calvino définit Ortes de la façon suivante : "C'era un uomo che voleva calcolare tutto" (C'était un homme qui désirait tout calculer). Italo Calvino, "Presentazione», dans Giammaria Ortes. Calcolo sopra la verità dell'istoria e altri scritti. Bartolo Anglani (éd.). Costa \& Nolan, Gênes, 1984

51. Giammaria Ortes, ibid., p. 135-148.

52. Ici je me range derrière l'opinion de Nino Valeri. II n'existe pas de preuves directes d'une telle influence, mais tout concourt pour qu'on la postule. Voir Nino Valeri, Pietro Verri, Florence, Felice Le Monnier, 1969., p. 201. (Studi e documenti di storia del risorgimento $\mathrm{XLIII)}$. 
53. Je conseille fortement la lecture de deux ouvrages incontournables. Premièrement il existe l'ouvrage désormais classique de Nino Valeri, Pietro Verri, op.cit,.et celle plus récente - mais également d'une très grande qualité - de Carlo Capra. I progressi della ragione. Vita di Pietro Verri (Les progrès de la raison. La vie de Pietro Verri).Bologne, II Mulino, 2002.

54. Voir de Gigliola di Renzo Villata, "Sembra que ...in genere...il mondo vada migliorando". Pietro Verri e la famiglia tra tradizione giuridica e innovazione» ("Il semble...en général...que le monde...va en s'améliorant". Pietro Verri et la famille entre la tradition juridique et l'innovation), dans Carlo Capra (dir.), Pietro Verri e il suo tempo (Pietro Verri et son temps). Actes d'un congrès tenu à Milan du 9 au 11 octobre 1997. Bologne, Cisalpino, 1999, tome I, p. 147-270. La relation épistolaire entre le père et le fils constitue un riche témoignage des rapports tendus qui existaient entre eux. Voir de Stefano Baia Curioni, Per sconfiggere l'oblio. Saggi e documenti sulla formazione intelletuale di Pietro Verri. (Pour vaincre l'oubli. Essais et documents sur la formation intellectuelle de Pietro Verri), Milan, Franco Angelli, 1988.

55. Lettre du 26 juillet 1763, dans Guido Sommi Picenardi, "Lettere inedite di Pietro Verri" (Lettres inédites de Pietro Verri), Rassegna Nazionale vol CLXXXXV, 1er juillet 1912 , p. 301-315, p. 315. Tant Venturi, que Valeri voient une valeur autobiographique dans le premier essai sur le bonheur de Verri. Voir Francesco Venturi, Settecento riformatore...op. cit., p. 661.

56. Pietro Verri aurait été vraisemblablement initié à la philosophie de Locke par Giambattista Roberti, son maître à Parme. Voir, Carlo Capra, I progressi..., op. cit., p. 99.

57. Prefazione al teatro comico del Sig.r Destouches (Préface au théâtre comique de Mr. Destouches) Reproduit dans Baia Curioni, Per sconfiggere, op. cit.

58. Voir Corrado Rosso, «Pietro Verri e la Francia : dall'lluminismo alla Rivoluzione» (Pietro Verri et la France : des Lumières à la révolution), dans Corrado Rosso, Felicità vo cercando: saggi in storia delle idee, Ravennes, Longo Editore, 1993, p. 91-118. (Speculum artium 18). À peine trois ans après sa parution en Italie, Gabriel Mingard en donna une traduction française. Pensées sur le bonheur, traduites de l'italien, Yverdon, 1766. (Je n'ai pu trouver cette traduction).

59. Voir de Gianni Francioni, «Metamorfosi della felicità. Dalle Meditazioni del 1763 al Discorso del 1781." (Les métamorphoses du bonheur. Des Méditations de 1763 au Discours de 1781), dans Carlo Capra (dir.), Pietro Verri, op. cit, p. 353-427.

60. Ce texte, écrit dans un temps relativement court, soit entre le 18 et le 24 octobre 1772, est apparu anonyme à Livourne en 1773 aux presses de L'Encyclopédie. II en existe une version comprenant des ajouts avec un titre différent, Discorso sull'indole del piacere e del dolore, Milan, Marelli. 1781. J'utilise la version parisienne de Molini, Opere filosofiche del comte Pietro Verri dell'Istituto delle Scienze di Bologna, 1781, regroupant en un seul volume les Meditazioni, les Idee et les Meditazioni sull'economia politica.

61. Alessandro, son frère, partage la même opinion que son aîné en disant des ldee sullindole del piacere : "Non ho letto niente di eguale in questo genere» (Je n'ai rien lu de pareil en son genre). Lettre du 11 novembre 1772.

62. "lo no dirò che il dolore per sè sia un bene, dirò bensì che il bene nasce dal male, la sterilità produce l'abbondanza, la povertà fa nascere la ricchezza, i bisogni 
Pietro Verri, théoricien milanais du bonheur ou La notion de rapidité résout-elle les problèmes liés au "bonheur froid et sec" de Maupertuis

concenti affinano l'ingegno, la somma ingiustizia fa nascere il coraggio, in una parola il dolore è il principio motore di tutto l'umano genere; egli è cagione di tutti i movimenti dell'uomo, che senza di lui sarebbe un animale inerte e stupido, e perirebbe poco dopo di esser nato"; Pietro Verri, Discorsi sull'indole...., p. 79.

63. II s'agit de l'épisode, rapporté dans le Phèdre, décrivant les sensations éprouvées par Socrate lorsqu'on lui a retiré ses fers.

64. II fait sienne, entre autres, la méthode lockienne de la genèse des idées (the way of ideas), puis il lui emprunte la notion d'inquiétude (uneasiness).

65. "ll tumulto dell'anima nel tempo d'una battaglia è sommo e tale, che dopo la vittoria si vedono gli uomini piú insensibili e induriti versare abbondanti lacrime di conzolazione, le quali il volgo le attribuirà ad affetto per il suo principe; ma il filosofo le conosce un effetto della cessazione d'un violento timore unito all'idea dei vantaggi personali che si sperano con questo nuovo grado di gloria acquistata." Lettre du 15 avril 1760. Cité dans Corrado Rosso, "Pietro Verri, "Il discorso sull'indole del piacere e del dolore" e Maupertuis", dans Corrado Rosso, Illuminismo, op. cit., p. 42-54.

66. À l'instar de Maupertuis qui mène, contre toute attente, son analyse géométrique des plaisirs et des douleurs vers une apologie du christianisme et une défense vibrante du cœur sur la raison, Verri se voit contraint d'abandonner l'objectif qu'il s'était fixé. II reconnaît qu'il ne peut trouver l'essence de la douleur et du plaisir. Son analyse minutieuse le mène vers un cul de sac. Par conséquent, il doit postuler l'existence d'un mystère pour expliquer comment on ressent la douleur ou du plaisir. On reconnait dans ses arguments un relent de vitalisme, probablement influencé en cela par le médecin et anatomiste Pietro Moscati (1739-1824).

67. "Egli così definì il piacere : il piacere è una sensazione, che l'uomo vuol piuttosto avere, che non avere. Questa però non è altrimenti una definizione, se ben vi si rifletta; sarebbe la stessa cosa il dire che il piacere è quel che piace : asserzione egualmente evidente quanto superflua..." II (Maupertuis) définit ainsi le plaisir : le plaisir est une sensation que l'âme aime mieux éprouver que ne pas éprouver. À bien y penser, cela n'est également pas une définition; cela reviendrait à dire que le plaisir est ce qui plaît; une assertion toute aussi évidente que redondante». Verri, Discorsi, op.cit., p. 5.

68. "Sono benaugurati sempre gli scritti, che fanno ripiegar l'uomo in sè medesimo, e l'obbligano a rendersi un esatto conto di ciò che sente. L'esame attento dei fenomeni interni è lo specchio della filosofia, e della morale umana." (Les œuvres qui font se replier les hommes sur eux-mêmes et les obligent à rendre minutieusement compte de ce qu'ils sentent sont toujours de bon augure. L'examen attentif des phénomènes internes est le miroir de la philosophie et de la morale humaine). Verri, Discorsi, op. cit., p 4.

69. En cela Verri reprend la distinction proposée par Maupertuis entre les plaisirs du corps et ceux de l'âme.

70. «l piaceri, e dolori morali sono tanto maggiori, quanto maggiore è il numero dei bisogni, e delle relazioni, che un uomo sente d'avere cogli altri.» Verri, Discorso, op. cit., p. 6.

71. "Tutte le sensazioni nostre piacevoli, o dolorose dipendono da tre soli principio : azione immediate sugli organi, speranza, e timore. II primo principio cagiona tutte le sensazioni fisiche; gli altri due le sensazioni morali.» Verri, Discorso, op. cit., p. 9 
72. «ll piacere nasce adunque dal dolore, e consiste nella rapida cessazione del dolore, ed è tanto maggiore, quanto lo fu il dolore, e più rapido l'annientamento di esso. Quanto più si diminuisce la rapidità, di tanto viene a scemarsi la sensazione piacevole nella energia.» Verri, Discorsi, op. cit., p. 24.

73. Verri, Discorsi, p. 48. Verri considère également le cas des douleurs innommées, c'est à dire des douleurs dont on ne peut localiser avec certitude l'emplacement exact. Verri donne comme exemple des maux de tête, des maux de dents, pour les plaisirs physiques, et l'ennui, l'inquiétude pour les plaisirs moraux. Verri postule l'existence de tels plaisirs afin de développer une théorie de l'origine des beauxarts. Voir, Piero Giordanetti, "Kant, Verri e le arti belle. Sulla fortuna di Verri in Germania.", dans Carlo Capra (dir. ) Pietro Verri, op. cit. p. 429-446.

74. Nous ne traiterons pas ici de la différence entre le processus physiologique de la douleur (ou du plaisir) et la perception que nous en avons (problème des qualias).

75. Considérons le cas suivant: J'ai un terrible mal de tête. On me donne des cachets pour soulager ma douleur. Puis, une fois l'effet des cachets disparu, j'ai de nouveau mal à la tête. Quelle est la durée de mon mal de tête? S'agit-il d'un seul mal de tête, ou devrions nous compter deux maux de tête? Si je considère que la durée de mon mal de tête s'étend du moment où je perçois une telle douleur jusqu'au moment où je ne la perçois plus du tout, en quoi puis-je être certain que je mesure toujours la durée du même mal de tête?

76. Je m'inspire ici vaguement de la théorie de Peter Geach quant aux propriétés intrinsèques et extrinsèques (ou relationnelles) de l'identité.

77. Je dédie ce texte à ma grande amie Claude Larue, qui suite à un accident de ski est demeurée clouée au lit pendant près de quatre mois. 